\title{
On two-dimensional ferromagnetism
}

\author{
Pablo Pedregal* $\quad$ Baisheng Yan $^{\dagger}$
}

\begin{abstract}
We study the existence of minimizers for 2-dimensional ferromagnetism with various assumptions on different terms of the total energy. Our general philosophy for finding the minimizing magnetizations is to reduce this problem to a minimization problem for a new energy over a divergence-free field. Such a general philosophy works for all dimensions. However, in the twodimensional case, since the divergence-free fields are equivalent to the rotated gradients, this new energy can be written as an integral functional of gradients and hence the minimization problem can be solved by some non-convex minimization procedures in the calculus of variations. We will apply this general philosophy to the two-dimensional case in this paper and to the three-dimensional situation in a future work. Special emphasis is placed on the analysis of existence/non-existence depending on the applied field $H$ and the physical domain $\Omega$.
\end{abstract}

\section{Introduction}

The model of micromagnetism seeks the magnetization $m: \Omega \subset \mathbf{R}^{N} \rightarrow \mathbf{R}^{N}$ of a body occupying the region $\Omega$ by minimizing the energy functional

$$
I(m)=\frac{\alpha}{2} \int_{\Omega}|\nabla m(x)|^{2} d x+\int_{\Omega} \varphi(m(x)) d x-\int_{\Omega} H \cdot m(x) d x+\frac{1}{2} \int_{\mathbf{R}^{N}}|F(z)|^{2} d z
$$

among all admissible magnetizations $m$ satisfying

$$
m \in L^{\infty}(\Omega), \quad|m(x)|=1 \text { a.e. } x \in \Omega,
$$

where $F \in L^{2}\left(\mathbf{R}^{N} ; \mathbf{R}^{N}\right)$ is the unique field determined by the simplified Maxwell's equations:

$$
\operatorname{curl} F=0, \quad \operatorname{div}\left(-F+m \chi_{\Omega}\right)=0 \text { in } \mathbf{R}^{N} .
$$

Here $\alpha>0$ is a material constant, $\varphi$ is the density of anisotropy that is minimized along preferred crystallographic directions, $H$ is a given external applied field (typically constant), and $F$ is the induced magnetic field on the whole $\mathbf{R}^{N}$ related to $m$ via Maxwell's equations (1.3). The first term

\footnotetext{
${ }^{*}$ ETSI Industriales, Universidad de Castilla-La Mancha, 13071 Ciudad Real, Spain (pablo.pedregal@uclm.es). Supported by project MTM2004-07114 from Ministerio de Educación y Ciencia (Spain) and by project PAI05-029 of JCCM (Castilla La Mancha).

${ }^{\dagger}$ Department of Mathematics, Michigan State University, East Lansing, MI 48824, USA (yan@math.msu.edu).
} 
in the energy $I(m)$ is called the exchange energy, the second term called the anisotropy energy, the third the external interaction energy, and the last term called the magnetostatic energy. There has been a considerable amount of work on this model exploring various possibilities, neglecting some of the terms over the others, studying limiting processes in various regimes $[9,10]$, looking at local minima [2], exploring existence over non-existence [12], etc. [13] is a recent comprehensive survey.

In the present paper, we are only concerned with existence of minimizers of the energy $I$ and their dependence on the given applied field under various sets of assumptions on exchange and anisotropy energies. We will not study the important issues on possible microstructure when minimizers do not exist or are not unique.

We will restrict attention to the two-dimensional case $N=2$, deferring the three-dimensional case to a future work. We will proceed in three-steps of increasing complexity.

1. The soft case where we neglect both the exchange energy and the anisotropy energy, and focus on the problem

$$
(P) \quad \text { Minimize in } m: \quad I(m)=\frac{1}{2} \int_{\mathbf{R}^{2}}|F(z)|^{2} d z-\int_{\Omega} H \cdot m(x) d x
$$

subject to

$$
\begin{gathered}
m \in L^{\infty}(\Omega), \quad|m(x)|=1 \text { a.e. } x \in \Omega, \\
\text { curl } F=0, \operatorname{div}\left(-F+m \chi_{\Omega}\right)=0 \text { in } \mathbf{R}^{2} .
\end{gathered}
$$

2. The hard case, where we consider the effect of a hard anisotropy, either uniaxial or cubic, but still neglect the exchange energy. In the unixial case, we will postulate anisotropy in the particular form

$$
\varphi(m)=\beta(1-|m \cdot e|),
$$

where $\beta>0$ and $e \in S^{1}$ is the unique preferred direction (easy axis); in the bi-axial or cubic case, we assume

$$
\varphi(m)=\min \left\{\beta_{1}\left(1-\left|m \cdot e_{1}\right|\right), \beta_{2}\left(1-\left|m \cdot e_{2}\right|\right)\right\},
$$

where $\beta_{1}, \beta_{2}>0$ and $e_{1}, e_{2} \in S^{1}$ are two independent preferred directions (easy axes). Here $\beta, \beta_{1}, \beta_{2}$ represent the strength of the anisotropy. Indeed we will treat the particular case where $e_{1}$ and $e_{2}$ are mutually orthogonal, and the strength of anisotropy is the same in both directions $\beta_{1}=\beta_{2}=\beta$.

3. The full case where we assume $\alpha>0$ and try to understand the interplay among the different contributions to the energy.

The main qualitative result concerning the existence of minimizers in all these cases can be summarized in the following theorem. More specific, quantitative results are given later.

Theorem 1.1 According to each case listed above, we have the following results.

1. In the soft case, $I(m)$ always has a minimizer for all domains $\Omega$ and all given fields $H$. 
2. In the hard case, there exists a proper, nonempty set $\mathcal{H} \subsetneq \mathbf{R}^{2}$ (depending on the domain $\Omega$ and the anisotropy density $\varphi$ ) such that $I(m)$ has a minimizer if $H \notin \mathcal{H}$ and does not have any minimizers if $H \in \mathcal{H}$; in the uniaxial case $0 \in \mathcal{H}$ and in the biaxial case $0 \notin \mathcal{H}$.

3. In the full case, $I(m)$ always has a minimizer.

Our general procedure to attack these problems is as follows, which is also valid regardless of dimension. First we introduce a new field $G=-F+m \chi_{\Omega}$ and a new augmented energy

$$
\begin{aligned}
\mathcal{A}(m, G) & =\frac{\alpha}{2} \int_{\Omega}|\nabla m|^{2}+\int_{\Omega} \varphi(m)-\int_{\Omega} H \cdot m+\frac{1}{2} \int_{\mathbf{R}^{N}}\left|m \chi_{\Omega}-G\right|^{2} \\
& =\int_{\Omega}\left[\frac{\alpha}{2}|\nabla m|^{2}+\varphi(m)-(H+G) \cdot m+\frac{1}{2}\left(|G|^{2}+1\right)\right]+\frac{1}{2} \int_{\Omega^{c}}|G|^{2} .
\end{aligned}
$$

We then define a new energy for $G \in L^{2}\left(\mathbf{R}^{N} ; \mathbf{R}^{N}\right)$ by putting

$$
J(G)=\inf _{\substack{m \in L^{\infty}(\Omega) \\|m|=1}} \mathcal{A}(m, G) .
$$

Here we do not distinguish the cases $\alpha=0$ and $\alpha>0$, but we use the convention that, for the admissible $m, \int_{\Omega}|\nabla m|^{2} d x=\infty$ unless $m \in H^{1}(\Omega)$. Therefore, if $\alpha>0, J(G)=\inf _{\substack{m \in H^{1}(\Omega) \\|m|=1}} \mathcal{A}(m, G)$.

The following easy facts establish our general philosophy that solving the minimization problem for energy $I$ over unit directions in $\Omega \subset \mathbf{R}^{N}$ is equivalent to solving the minimization problem for the new energy $J$ over divergence-free fields on $\mathbf{R}^{N}$. We will apply this general philosophy to the two-dimensional case in this paper and to the three-dimensional situation in a future work.

Proposition 1.2 (i) For any $m \in L^{\infty}(\Omega)$ with $|m(x)|=1$ a.e.,

$$
I(m)=\min _{\substack{G \in L^{2}\left(\mathbf{R}^{N} ; \mathbf{R}^{N}\right) \\ \operatorname{div} G=0}} \mathcal{A}(m, G) .
$$

Moreover, if $I(m)<\infty$, then the minimizer $G=G_{m}$ is unique and satisfies

$$
\operatorname{div} G_{m}=0, \quad \operatorname{curl}\left(m \chi_{\Omega}-G_{m}\right)=0 ;
$$

hence $F=F_{m}=m \chi_{\Omega}-G_{m}$ is the unique solution to Maxwell's equations (1.3) above.

(ii) For any $G \in L^{2}\left(\mathbf{R}^{N} ; \mathbf{R}^{N}\right), J(G)$ is attained by minimizers; that is,

$$
J(G)=\min _{\substack{m \in L \infty(\Omega) \\|m|=1}} \mathcal{A}(m, G) .
$$

We denote by $\Sigma(G)$ the set of all these minimizers.

(iii) It follows that

$$
\inf _{\substack{m \in L^{\infty}(\Omega) \\|m|=1}} I(m)=\inf _{\substack{G \in L^{2}\left(\mathbf{R}^{N} ; \mathbf{R}^{N}\right) \\ \operatorname{div} G=0}} J(G) .
$$

Moreover, if $\bar{m} \in L^{\infty}(\Omega),|\bar{m}|=1$, is a minimizer of $I$ then the function $G_{\bar{m}}$ determined by the Maxwell equation (1.9) above is a minimizer of $J ;$ if $\bar{G} \in L^{2}\left(\mathbf{R}^{N} ; \mathbf{R}^{N}\right), \operatorname{div} \bar{G}=0$, is a minimizer of $J$ then any function $\tilde{m} \in \Sigma(\bar{G})$ is a minimizer of $I$. 
Proof. The proof of this proposition is straightforward. It shows that finding optimal magnetic configurations amounts, after all, to minimizing the energy $\mathcal{A}(m, G)$ recursively on $m$ or on $G$, depending on what it might be more convenient. The main observation here is that for given $m$, the minimization in divergence-free vector fields $G$ takes place for

$$
\frac{1}{2} \int_{\mathbf{R}^{N}}\left|m \chi_{\Omega}-G\right|^{2}
$$

It is well-known that the unique solution is determined by the Helmhotz projection

$$
\operatorname{div} G=0 \quad \operatorname{curl}\left(m \chi_{\Omega}-G\right)=0 .
$$

On the other hand for $G$ fixed, the minimization on $m$ is a regular variational problem for $\alpha>0$ (coercive and convex). If $\alpha=0$, then optimal fields $m$ arise by pointwise minimizing the integrand

$$
\min _{|m|=1}(\varphi(m)-(H+G) m) .
$$

Part (iii) is a direct consequence of parts (i) and (ii).

The case involving the derivative of field $m$ presents an easier problem regarding the existence of minimizers by virtue of the following result, although the concrete computations of minimizers seem a much harder nonlocal problem $[2,6]$.

Proposition 1.3 Let $\alpha>0$. Then the energy $J$ defined above is sequentially weakly lower semicontinous and coercive on $L^{2}\left(\mathbf{R}^{N} ; \mathbf{R}^{N}\right)$, and hence $J$ possesses a minimizer over the divergence-free fields; therefore, the energy I has minimizers $\bar{m} \in \Sigma(\bar{G})$, where $\bar{G}$ is any minimizer of J. Furthermore, any such pair $(\bar{m}, \bar{G})$ is also a minimizer for the augmented energy $\mathcal{A}$ defined above.

Proof. Assume $G_{j} \rightarrow G_{0}$ weakly in $L^{2}\left(\mathbf{R}^{N} ; \mathbf{R}^{N}\right)$. By Proposition 1.2 (ii) above, there exist minimizers $m_{j} \in L^{\infty}(\Omega)$ with $\left|m_{j}(x)\right|=1$ a.e. such that

$$
J\left(G_{j}\right)=\mathcal{A}\left(m_{j}, G_{j}\right) \leq M, \quad j=0,1, \cdots,
$$

where $M<\infty$ is some constant. Since $\alpha>0$, it follows that $m_{j} \in H^{1}(\Omega)$ and $\left\|\nabla m_{j}\right\|_{L^{2}(\Omega)} \leq M^{\prime}$ for another constant $M^{\prime}$. We can assume, without loss of generality, that $m_{j} \rightarrow m^{*}$ weakly in $H^{1}(\Omega)$ and $m_{j}(x) \rightarrow m^{*}(x)$ and hence $\left|m^{*}(x)\right|=1$ for almost every $x \in \Omega$. Note that

$$
\mathcal{A}\left(m_{j}, G_{j}\right)=\frac{\alpha}{2} \int_{\Omega}\left|\nabla m_{j}\right|^{2}+\int_{\Omega} \varphi\left(m_{j}\right)-\int_{\Omega} H \cdot m_{j}+\frac{1}{2} \int_{\mathbf{R}^{N}}\left|m_{j} \chi_{\Omega}-G_{j}\right|^{2} .
$$

Taking the liminf as $j \rightarrow \infty$, we have

$$
\begin{aligned}
\liminf _{j \rightarrow \infty} J\left(G_{j}\right) & =\liminf _{j \rightarrow \infty} \mathcal{A}\left(m_{j}, G_{j}\right) \\
& \geq \frac{\alpha}{2} \int_{\Omega}\left|\nabla m^{*}\right|^{2}+\int_{\Omega} \varphi\left(m^{*}\right)-\int_{\Omega} H \cdot m^{*}+\frac{1}{2} \int_{\mathbf{R}^{N}}\left|m^{*} \chi_{\Omega}-G_{0}\right|^{2} \\
& =\mathcal{A}\left(m^{*}, G_{0}\right) \geq J\left(G_{0}\right) .
\end{aligned}
$$

This proves the lower semicontinuity of $J$ on $L^{2}\left(\mathbf{R}^{N} ; \mathbf{R}^{N}\right)$. Moreover, it follows easily that (even when $\alpha=0$ )

$$
J(G) \geq \frac{1}{4}\|G\|_{L^{2}\left(\mathbf{R}^{N} ; \mathbf{R}^{N}\right)}^{2}-C,
$$


where $C$ is a constant depending only on $\varphi$ and $H$. This proves the coercivity of $J$. The rest of the proof follows easily.

Remark. If dimension $N=2$, we can use the angle as an independent variable to represent the direction of magnetization $m$ as

$$
m=(\cos \theta, \sin \theta), \quad \theta: \Omega \rightarrow \mathbf{R} .
$$

Notice that $|\nabla m|^{2}=|\nabla \theta|^{2}$. If we introduce $\mathbf{v}=\left(v_{1}, v_{2}\right)=(\theta, u)$, where $G=\nabla^{\perp} u$, then the augmented energy $\mathcal{A}(m, G)$ above can be written as a variational integral:

$$
\mathcal{A}(m, G)=\mathcal{E}(\mathbf{v})=\int_{\mathbf{R}^{2}} W(x, \mathbf{v}(x), \nabla \mathbf{v}(x)) d x,
$$

where $W(x, \mathbf{v}, A)$ is a function of $x, \mathbf{v} \in \mathbf{R}^{2}$ and $2 \times 2$ matrix $A$ defined by

$$
W(x, \mathbf{v}, A)=\frac{1}{2}\left|A_{2}\right|^{2}+\chi_{\Omega}(x)\left[\varphi\left(\cos v_{1}, \sin v_{1}\right)-\left(H+A_{2}^{\perp}\right) \cdot\left(\cos v_{1}, \sin v_{1}\right)+\frac{\alpha}{2}\left|A_{1}\right|^{2}\right]
$$

with $A_{1}, A_{2}$ denoting the first and second row of matrix $A$. In fact, if $\alpha>0$, this density function $W(x, \mathbf{v}, A)$ is convex in $A$, and hence the minimization problem in this case can be solved easily by the standard direct method of the calculus of variations.

The rest of discussions is devoted to the case when there is no exchange energy. Again our discussion is valid regardless of dimension. Let $\alpha=0$ and write

$$
\mathcal{A}(m, G)=\int_{\Omega} \rho(m(x), G(x)) d x+\frac{1}{2} \int_{\Omega^{c}}|G(z)|^{2} d z ; \quad \Omega^{c}=\mathbf{R}^{N} \backslash \Omega,
$$

where

$$
\rho(h, \xi)=\varphi(h)-(H+\xi) \cdot h+\frac{1}{2}\left(|\xi|^{2}+1\right) .
$$

Define the function

$$
\Psi(\xi)=\min _{h \in S^{N-1}} \rho(h, \xi)=\min _{h \in S^{N-1}}\left[\varphi(h)-(H+\xi) \cdot h+\frac{1}{2}\left(|\xi|^{2}+1\right)\right]
$$

and its minimal set

$$
\sigma(\xi)=\left\{h \in S^{N-1} \mid \rho(h, \xi)=\Psi(\xi)\right\} .
$$

This coming proposition is a more explicit way of writing the previous one for the case $\alpha=0$.

Proposition 1.4 Let $\alpha=0$. Then the energy $J(G)$ defined above is given by

$$
J(G)=\int_{\Omega} \Psi(G(x)) d x+\frac{1}{2} \int_{\Omega^{c}}|G(z)|^{2} d z=\mathcal{A}\left(m_{G}, G\right),
$$

where $m_{G} \in L^{\infty}(\Omega)$ is any function satisfying

$$
m_{G}(x) \in \sigma(G(x)) \quad \text { a.e. } \quad x \in \Omega ;
$$

all such functions $m_{G}$ constitute the solution set $\Sigma(G)$ defined in Proposition 1.2. 
We now discuss our study in the two-dimensional case. Assume dimension $N=2$. Then every divergence-free field $G$ can be written as a rotated gradient:

$$
G(x)=T \nabla u(x)=\nabla^{\perp} u(x),
$$

where $\nabla u(x)=\left(u_{x_{1}}, u_{x_{2}}\right)$ and $T(a, b)=(a, b)^{\perp}=(-b, a)$. Therefore, if $\alpha=0$, the energy $J(G)$ can be written as a variational integral

$$
J(G)=E(u)=\int_{\mathbf{R}^{2}} \Phi(x, \nabla u(x)) d x,
$$

where $u: \mathbf{R}^{2} \rightarrow \mathbf{R}$ is measurable with distributional gradient $\nabla u$ belonging to $L^{2}\left(\mathbf{R}^{2} ; \mathbf{R}^{2}\right)$ and

$$
\Phi(x, \lambda)=\chi_{\Omega}(x) \psi(\lambda)+\chi_{\Omega^{c}}(x) \frac{1}{2}|\lambda|^{2}, \quad \lambda \in \mathbf{R}^{2},
$$

where $\psi(\lambda)=\Psi\left(\lambda^{\perp}\right)$.

Proposition 1.5 Let $N=2$ and $\alpha=0$. Then any minimizer $\bar{u}$ of $E$ gives a minimizer $\bar{G}$ of $J$ in terms of $\bar{G}=\nabla^{\perp} u$, and hence gives a minimizer $\bar{m}$ of I in terms of $\bar{m}(x) \in \sigma\left(\nabla^{\perp} u(x)\right)$ for almost every $x \in \Omega$.

It is interesting to notice that, in some cases, one can compute explicitly the density $\psi(\lambda)=\Psi\left(\lambda^{\perp}\right)$. In fact, in the soft case $\varphi \equiv 0$, then one can find easily that

$$
\psi(\lambda)=\frac{1}{2}\left(\left|\lambda-H^{\perp}\right|-1\right)^{2}-\frac{1}{2}|H|^{2}+\lambda \cdot H^{\perp} .
$$

In the uniaxial case with $e$ pointing in the easy direction, one can find

$$
\psi(\lambda)=\frac{1}{2}\left(|\lambda|^{2}+1\right)+\beta-\sqrt{\left|\lambda-H^{\perp}\right|^{2}+2 \beta\left|\left(\lambda-H^{\perp}\right) \cdot e^{\perp}\right|+\beta^{2}},
$$

where $\beta>0$ is the parameter associated with the strength of anisotropy. A similar expression can be given in the biaxial case (see Section 4.2).

The analysis of the corresponding variational problems leads to a scalar, nonconvex problem for certain integral functionals of gradients on the whole space. In Section 2, we study a general variational principle which explicitly works for such functionals on the whole space. In this setting, some existence results (e.g., [3, 4, 5, 7, 8, 19]) under lack of convexity can nevertheless be applied. Then we apply this general principle to the soft and hard cases of two-dimensional ferromagnetism in Sections 3 and 4. In the soft case, we have the existence of minimizers for all domains and all applied fields (Section 3), and some times we even have explicit solutions. In the hard case, we have results on non-existence of minimizers in the uniaxial case for some applied fields $H$ (this was already very well-known [12]) and the existence of minimizers for other $H$, and the similar results in the cubic (biaxial) case (Section 4). In the case of a unit disk, we have a rather explicit characterization of the set $\mathcal{H}$. 


\section{A general variational principle}

Though the minimization problem in (1.16) is equivalent to the micromagnetics functional only for dimension $N=2$, it is worth analyzing such functional regardless of dimension. We therefore focus in this section on the minimization problem for variational functionals of the type as defined by (1.16); that is,

$$
E(u)=\int_{\mathbf{R}^{N}} \Phi(x, \nabla u(x)) d x=\int_{\Omega} \psi(\nabla u(x)) d x+\frac{1}{2} \int_{\Omega^{c}}|\nabla u(x)|^{2} d x,
$$

where $N$ is any dimension, $\Omega \subset \mathbf{R}^{N}$ is a bounded domain with piece-wise smooth boundary and $\psi: \mathbf{R}^{N} \rightarrow \mathbf{R}$ is a locally Lipschitz function satisfying, for some constants $c_{2} \geq c_{0}>0, c_{1}, c_{3} \in \mathbf{R}$,

$$
c_{0}|\lambda|^{2}-c_{1} \leq \psi(\lambda) \leq c_{2}|\lambda|^{2}+c_{3}, \quad \lambda \in \mathbf{R}^{N} .
$$

The natural admissible class for $E(u)$ is the space of functions $u \in H_{l o c}^{1}\left(\mathbf{R}^{N}\right)$ whose gradient $\nabla u$ belongs to $L^{2}\left(\mathbf{R}^{N} ; \mathbf{R}^{N}\right)$; in this case, the functional $E$ is finite-valued. Also, it is easy to see that $E(u)=E(u+c)$ for all constants $c \in \mathbf{R}$. Note that for all $u \in H_{l o c}^{1}\left(\mathbf{R}^{N}\right)$ the trace $\Gamma u=\left.u\right|_{\partial \Omega}$ is well defined and, by the trace theorem [1], the trace operator $\Gamma: H_{l o c}^{1}\left(\mathbf{R}^{N}\right) \rightarrow H^{1 / 2}(\partial \Omega)$ is onto; moreover, $\Gamma$ is also compact from $H_{l o c}^{1}\left(\mathbf{R}^{N}\right)$ into $L^{2}(\partial \Omega)$.

We introduce the following function spaces:

$$
\begin{gathered}
X=\left\{u \in H_{l o c}^{1}\left(\mathbf{R}^{N}\right) \mid \nabla u \in L^{2}\left(\mathbf{R}^{N} ; \mathbf{R}^{N}\right), \int_{\partial \Omega} \Gamma u(x) d S=0\right\}, \\
Y=\Gamma(X)=\left\{g \in H^{1 / 2}(\partial \Omega) \mid \int_{\partial \Omega} g d S=0\right\} .
\end{gathered}
$$

Define spaces $X_{1}, X_{2}$ to be the restriction of $X$ on $\Omega$ and $\Omega^{c}$, respectively. Note that if $u_{i} \in X_{i}$ $(i=1,2)$ satisfy $\left.u_{1}\right|_{\partial \Omega}=\left.u_{2}\right|_{\partial \Omega}$, then the function $u=\chi_{\Omega} u_{1}+\chi_{\Omega^{c}} u_{2} \in X$.

We study the minimization problem for functional $E$ on the space $X$. To this end, we define the relaxation functional of $E$ by

$$
E^{\#}(u)=\int_{\Omega} \psi^{\#}(\nabla u(x)) d x+\frac{1}{2} \int_{\Omega^{c}}|\nabla u(x)|^{2} d x,
$$

where $\psi^{\#}$ is the convexification of $\psi$.

The following result establishes that the standard relaxation theorem still holds in this case where $E(u)$ is not a standard variational integral on bounded domains.

Proposition 2.1 It follows that

$$
\inf _{u \in X} E(u)=\min _{u \in X} E^{\#}(u)
$$

Proof. Since $E$ is not a standard variational integral defined on bounded domains, we include the proof for completeness of the paper. We show first that $\inf _{X} E=\inf _{X} E^{\#}$ and then that the infimum of $E^{\#}$ is attained. Assume $\inf _{X} E^{\#}<\infty$. Given any $\epsilon>0$, let $u_{\epsilon} \in X$ be such that $E^{\#}\left(u_{\epsilon}\right) \leq \inf _{X} E^{\#}+\epsilon$. Let $g_{\epsilon}=\Gamma u_{\epsilon}=\left.u_{\epsilon}\right|_{\partial \Omega} \in Y$. By the standard relaxation theorem [15],

$$
\inf _{v \in H^{1}(\Omega),\left.v\right|_{\partial \Omega}=g_{\epsilon}} \int_{\Omega} \psi(\nabla v(x)) d x=\inf _{v \in H^{1}(\Omega),\left.v\right|_{\partial \Omega}=g_{\epsilon}} \int_{\Omega} \psi^{\#}(\nabla v(x)) d x .
$$


Hence there exists $v_{\epsilon} \in H^{1}(\Omega)$ with $\left.v_{\epsilon}\right|_{\partial \Omega}=g_{\epsilon}$ such that

$$
\int_{\Omega} \psi\left(\nabla v_{\epsilon}\right) d x \leq \inf _{v \in H^{1}(\Omega),\left.v\right|_{\partial \Omega}=g_{\epsilon}} \int_{\Omega} \psi^{\#}(\nabla v) d x+\epsilon \leq \int_{\Omega} \psi^{\#}\left(\nabla u_{\epsilon}\right) d x+\epsilon .
$$

Let $\tilde{u}=\chi_{\Omega} v_{\epsilon}+\chi_{\Omega^{c}} u_{\epsilon}$. Then $\tilde{u} \in X$ and

$$
\begin{aligned}
E(\tilde{u}) & =\int_{\Omega} \psi\left(\nabla v_{\epsilon}\right) d x+\frac{1}{2} \int_{\Omega^{c}}\left|\nabla u_{\epsilon}\right|^{2} d x \\
& \leq \int_{\Omega} \psi^{\#}\left(\nabla u_{\epsilon}\right) d x+\epsilon+\frac{1}{2} \int_{\Omega^{c}}\left|\nabla u_{\epsilon}\right|^{2} d x \\
& =E^{\#}\left(u_{\epsilon}\right)+\epsilon \leq \inf _{X} E^{\#}+2 \epsilon .
\end{aligned}
$$

From this it follows that $\inf _{X} E \leq \inf _{X} E^{\#}$. Hence $\inf _{X} E=\inf _{X} E^{\#}$. To show that $E^{\#}$ has a minimizer on $X$, we use the standard direct method. Let $\left\{u_{j}\right\}$ be a minimizing sequence of $E^{\#}$. Then $\left\{\nabla u_{j}\right\}$ is a bounded sequence in $L^{2}\left(\mathbf{R}^{N} ; \mathbf{R}^{N}\right)$. Hence assume (via a subsequence) $\nabla u_{j} \rightarrow U$ weakly in $L^{2}\left(\mathbf{R}^{N} ; \mathbf{R}^{N}\right)$ for some function $U \in L^{2}\left(\mathbf{R}^{N} ; \mathbf{R}^{N}\right)$. The gradient structure of $\nabla u_{j}$ also implies that $U$ is a gradient; that is, $U=\nabla \bar{u}$ for some function $\bar{u} \in H_{l o c}^{1}\left(\mathbf{R}^{N}\right)$. On the other hand, since $\int_{\partial \Omega} \Gamma u_{j} d S=0$, it follows that $\left\{u_{j}\right\}$ is a bounded sequence in $H^{1}(\Omega)$. Hence (also via a subsequence) assume $u_{j} \rightarrow \bar{v}$ weakly in $H^{1}(\Omega)$. The compactness of the trace operator also implies $\int_{\partial \Omega} \Gamma \bar{v} d S=0$. Since $\nabla \bar{u}=\nabla \bar{v}$ in $\Omega$, it follows that $\bar{u}(x)=\bar{v}(x)+c$ for almost every $x \in \Omega$ and a constant $c$. Therefore $\tilde{u}=\bar{u}-c \in X$ and from the lower semicontinuities of both parts of $E^{\#}(u)$ it follows easily that $E^{\#}(\tilde{u}) \leq \liminf E^{\#}\left(u_{j}\right)=\inf _{X} E^{\#}$. Hence $\tilde{u} \in X$ is a minimizer of $E^{\#}$ on $X$.

To characterize the minimizers of $E^{\#}$, we first have the following exterior uniqueness result.

Proposition 2.2 Let $u_{1}, u_{2}$ be any two minimizers of $E^{\#}$ on $X$. Then $u_{1}=u_{2}$ on $\Omega^{c}$. Therefore, there exists a unique $\bar{g} \in Y$ such that all minimizers $\bar{u}$ of $E^{\#}$ satisfy $\Gamma \bar{u}=\left.\bar{u}\right|_{\partial \Omega}=\bar{g}$.

Proof. We write $E^{\#}(u)=P_{1}(u)+P_{2}(u)$, where

$$
P_{1}(u)=\int_{\Omega} \psi^{\#}(\nabla u(x)) d x, \quad P_{2}(u)=\frac{1}{2} \int_{\Omega^{c}}|\nabla u(x)|^{2} d x .
$$

Let $f(t)=E^{\#}\left(t u_{1}+(1-t) u_{2}\right)$ and $p_{i}(t)=P_{i}\left(t u_{1}+(1-t) u_{2}\right)(i=1,2)$ for $0 \leq t \leq 1$. Then all these functions are convex on $[0,1]$ and $f(t)=p_{1}(t)+p_{2}(t)$. Moreover $f(0)=f(1)=\min _{[0,1]} f$. From this it follows easily that $f(t)=f(0)$ for all $t \in[0,1]$. Since $p_{i}(t) \leq t p_{i}(1)+(1-t) p_{i}(0)$, $(i=1,2)$, the equality $f(t)=f(0)=f(1)$ implies that $p_{i}(t)=t p_{i}(1)+(1-t) p_{i}(0)$ for $i=1,2$. In particular, $p_{2}\left(\frac{1}{2}\right)=\frac{1}{2}\left(p_{2}(0)+p_{2}(1)\right)$. This equality implies $\nabla u_{1}=\nabla u_{2}$ on $\Omega^{c}$. Hence $u_{1}=u_{2}$ on $\Omega^{c}$ since $\int_{\partial \Omega} \Gamma u_{i} d S=0$ for $i=1,2$. In particular, $\left.u_{1}\right|_{\partial \Omega}=\left.u_{2}\right|_{\partial \Omega}$. This proves the proposition.

If $\psi^{\#}$ is $C^{1}$, the minimizers of $E^{\#}$ can be exactly solved as the weak solutions of the Euler-Lagrange equation for $E^{\#}$. From this, we have the following result characterizing the minimizers of $E^{\#}$ and hence the boundary data $\bar{g}$.

Theorem 2.3 Let $\psi^{\#}$ be $C^{1}$. Then, $\bar{u}=\chi_{\Omega} \bar{v}+\chi_{\Omega^{c}} \bar{w}$ is a minimizer of $E^{\#}$ if and only if $\bar{v}, \bar{w}$ 
satisfy the following conditions:

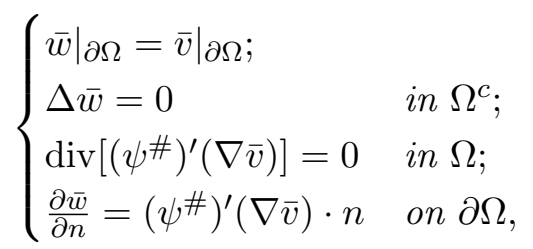

where $n(x)$ is the unit outward normal to the boundary $\partial \Omega$ of the interior domain $\Omega$.

Proof. Since $E^{\#}$ is convex, the minimizers of $E^{\#}$ are exactly the weak solutions of the EulerLagrange equation. Therefore, $\bar{u}=\chi_{\Omega} \bar{v}+\chi_{\Omega^{c}} \bar{w}$ is a minimizer of $E^{\#}$ if and only if

$$
\int_{\Omega}\left(\psi^{\#}\right)^{\prime}(\nabla \bar{v}) \cdot \nabla \zeta d x+\int_{\Omega^{c}} \nabla \bar{w} \cdot \nabla \zeta d x=0, \quad \forall \zeta \in X .
$$

Clearly, this condition is equivalent to (2.4).

Remark. Certainly, (2.4) is a difficult condition involving the mixed Dirichlet-Neumann boundary conditions and depending heavily on the structures of function $\psi^{\#}$ and domain $\Omega$; nevertheless, it always has solutions. Also, from Proposition 2.2, although the solution $\bar{v}$ to (2.4) may not be unique, the solution $\bar{w}$ and hence the boundary trace $\bar{g}=\left.\bar{w}\right|_{\partial \Omega}=\left.\bar{v}\right|_{\partial \Omega}$ must be unique.

Using the unique boundary data $\bar{g}$, we can derive a necessary and sufficient condition for minimizers of $E^{\#}$.

Theorem 2.4 Let $\bar{g} \in Y$ be the unique boundary data determined in the previous proposition. Then $\bar{u} \in X$ is a minimizer of $E^{\#}$ on $X$ if and only if $\bar{u}=\chi_{\Omega} \bar{v}+\chi_{\Omega^{c}} \bar{w}$, where $\bar{w}=\omega(\bar{g})$ is the unique solution to the Dirichlet problem:

$$
\Delta \bar{w}=0 \quad \text { in } \Omega^{c},\left.\quad \bar{w}\right|_{\partial \Omega}=\bar{g},
$$

and $\bar{v}$ is any minimizer of the following problem:

$$
\int_{\Omega} \psi^{\#}(\nabla \bar{v}) d x=\min _{v \in H^{1}(\Omega),\left.v\right|_{\partial \Omega}=\bar{g}} \int_{\Omega} \psi^{\#}(\nabla v) d x .
$$

Proof. First, assume $\bar{u} \in X$ is a minimizer of $E^{\#}$. Then, by Proposition 2.2 above, $\left.\bar{u}\right|_{\partial \Omega}=\bar{g}$. Let $E^{\#}(u)=P_{1}(u)+P_{2}(u)$ with $P_{i}$ introduced in the previous proof. Since $E^{\#}(\bar{u}) \leq E^{\#}(\bar{u}+\zeta)$ for all $\zeta \in X$, it follows by choosing $\zeta=0$ on $\Omega^{c}$ or on $\Omega$ that $\bar{u}$ minimizes each $P_{i}$ with the given boundary data $\bar{g}$. The minimizer of $P_{2}$ must be harmonic and given by the unique solution $\bar{w}=\omega(\bar{g})$ of the Dirichlet problem (2.6) above. Therefore, $\bar{u}=\chi_{\Omega} \bar{v}+\chi_{\Omega^{c}} \omega(\bar{g})$, where $\bar{v} \in X_{1}$ is a minimizer of problem (2.7). Now, assume $\bar{u}=\chi_{\Omega} \bar{v}+\chi_{\Omega^{c}} \bar{w}$, where $\bar{v} \in X_{1}$ is a minimizer of problem (2.7) and $\bar{w}=\omega(\bar{g})$ is the solution of the Dirichlet problem (2.6) above. We want to show $E^{\#}(\bar{u})=\min _{X} E^{\#}$. Let $\tilde{u} \in X$ be a minimizer of $E^{\#}$ on $X$; that is,

$$
\min _{X} E^{\#}=E^{\#}(\tilde{u})=\int_{\Omega} \psi^{\#}(\nabla \tilde{u}) d x+\frac{1}{2} \int_{\Omega^{c}}|\nabla \tilde{u}|^{2} d x
$$


Since $\left.\tilde{u}\right|_{\partial \Omega}=\bar{g}$, by the assumptions of $\bar{v}$ and $\bar{w}$, the first term on the right-hand side of this equation is no less than $\int_{\Omega} \psi^{\#}(\nabla \bar{v}) d x$ and the second term is no less than $\frac{1}{2} \int_{\Omega^{c}}|\nabla \bar{w}|^{2} d x$. Hence $E^{\#}(\tilde{u}) \geq E^{\#}(\bar{u})$. This shows that $E^{\#}(\bar{u})=E^{\#}(\tilde{u})=\min _{X} E^{\#}$.

We now give the necessary and sufficient condition for existence of minimizers of the original functional $E(u)$.

Theorem 2.5 Let $\bar{g} \in Y$ be the unique boundary data determined in Proposition 2.2 above. Then, the functional $E$ has a minimizer over $X$ if and only if the minimization problem

$$
\inf _{v \in H^{1}(\Omega),\left.v\right|_{\partial \Omega}=\bar{g}} \int_{\Omega} \psi(\nabla v(x)) d x
$$

has a minimizer. Moreover, a function $\bar{u} \in X$ is a minimizer of $E$ if and only if

$$
\bar{u}=\chi_{\Omega} \tilde{v}+\chi_{\Omega^{c}} \omega(\bar{g}),
$$

where $\tilde{v}$ is any minimizer of problem (2.8).

Corollary 2.6 $\bar{u} \in X$ is a minimizer of $E$ if and only if $\bar{u}$ is a minimizer of $E^{\#}$ and

$$
\psi(\nabla \bar{u}(x))=\psi^{\#}(\nabla \bar{u}(x)) \quad \forall x \in \Omega .
$$

Furthermore, if $\psi^{\#}$ is $C^{1}$ and $\bar{u}=\chi_{\Omega} \bar{v}+\chi_{\Omega^{c}} \bar{w}$, then $\bar{u}$ is a minimizer of $E$ if and only if $\bar{v}, \bar{w}$ satisfy the following conditions:

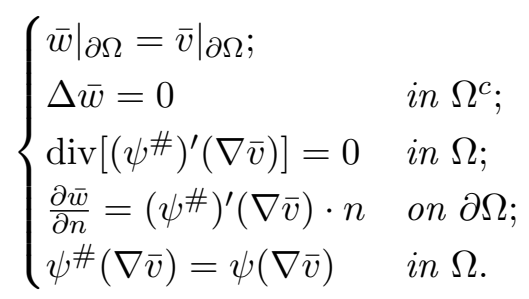

Remark. If both $\psi, \psi^{\#}$ are $C^{1}$, then any function $\bar{u}$ satisfying condition (2.11) must be a weak solution of the Euler-Lagrange equation for the functional $E(u)$. To see this, we simply note that in this case $\left(\psi^{\#}\right)^{\prime}\left(\lambda_{0}\right)=\psi^{\prime}\left(\lambda_{0}\right)$ whenever $\psi^{\#}\left(\lambda_{0}\right)=\psi\left(\lambda_{0}\right)$. However, as in most nonconvex problems, the Euler-Lagrange equation for $E(u)$ alone only provides a necessary condition for possible minimizers of $E(u)$, but it is far away from being sufficient for the existence of minimizers. When the condition $\psi(\nabla \bar{u})=\psi^{\#}(\nabla \bar{u})$ a.e. in $\Omega$ is not satisfied by any minimizers $\bar{u}$ of $E^{\#}$, the (nonconvex) functional $E$ does not have any minimizers.

The following result provides a sufficient condition for existence of minimizers of $E$.

Corollary 2.7 Assume $\psi^{\#}$ is affine on each component of the detachment set $\mathcal{D}=\left\{\lambda \mid \psi^{\#}(\lambda)<\right.$ $\psi(\lambda)\}$. Then the energy $E$ has minimizers. 
Proof. Under the given condition, we can easily see that the conditions of [3, Theorem 2.1] are all satisfied. This implies that the functional

$$
F(v)=\int_{\Omega} \psi(\nabla v(x)) d x
$$

itself has minimizers for all boundary data $g$ (see also [19]). Let $\tilde{v} \in X_{1}$ be any minimizer of $F$ for the unique boundary data $\bar{g}$ determined above. Then, by Theorem 2.5 , the function

$$
\tilde{u}=\chi_{\Omega} \tilde{v}+\chi_{\Omega^{c}} \omega(\bar{g})
$$

is a minimizer of $E$.

Finally we have an explicit result in the case when domain $\Omega$ is the unit ball.

Theorem 2.8 Let $\Omega=B$ be the unit ball in $\mathbf{R}^{N}$. Then

$$
\inf _{X} E=\min _{X} E^{\#}=|B| \min _{\lambda \in \mathbf{R}^{N}}\left(\psi^{\#}(\lambda)+\frac{N-1}{2}|\lambda|^{2}\right)=|B|\left(\psi^{\#}(\bar{\lambda})+\frac{N-1}{2}|\bar{\lambda}|^{2}\right),
$$

where $\bar{\lambda} \in \mathbf{R}^{N}$ is uniquely determined. In this case, the minimizers of $E^{\#}$ are given by $\bar{u}=$ $\chi_{B}(x) \bar{v}(x)+\chi_{B^{c}}(x) \frac{\bar{\lambda} \cdot x}{|x|^{N}}$, where $\bar{v}$ is any function satisfying

$$
\left.\bar{v}\right|_{\partial B}=\bar{\lambda} \cdot x, \quad \int_{B} \psi^{\#}(\nabla \bar{v}(x)) d x=|B| \psi^{\#}(\bar{\lambda}) .
$$

Furthermore, $E$ has a minimizer if and only if either $\psi(\bar{\lambda})=\psi^{\#}(\bar{\lambda})$ or

$$
\bar{\lambda} \in \operatorname{intconv}\left\{\lambda_{1}, \lambda_{2}, \cdots, \lambda_{k}\right\} \quad \text { (the interior of convex hull of } \lambda_{1}, \cdots, \lambda_{k} \text { ) }
$$

for some $\lambda_{j} \in \mathbf{R}^{N}, j=1,2, \cdots, k$, satisfying $\cap_{j=1}^{k} \partial \psi\left(\lambda_{j}\right) \neq \emptyset$.

Remark. (i) If $\psi^{\#}$ is $C^{1}$ then $\bar{\lambda}$ is uniquely determined by the algebraic equation

$$
\left(\psi^{\#}\right)^{\prime}(\bar{\lambda})+(N-1) \bar{\lambda}=0 .
$$

(ii) The last condition $\cap_{j=1}^{k} \partial \psi\left(\lambda_{j}\right) \neq \emptyset$ is equivalent to that $\psi^{\#}$ be affine on the convex hull $\operatorname{conv}\left\{\lambda_{1}, \lambda_{2}, \cdots, \lambda_{k}\right\}$.

We need the following elementary result for proving this theorem.

Lemma 2.9 Let $f$ and $g$ be two functions on $\mathbf{R}^{N}$ and let $f$ be convex. Assume $f+g$ has a local minimum at $\lambda_{0}$ and $g$ is differentiable at $\lambda_{0}$. Then it follows that

$$
f\left(\lambda_{0}+\eta\right) \geq f\left(\lambda_{0}\right)-g^{\prime}\left(\lambda_{0}\right) \cdot \eta, \quad \forall \eta \in \mathbf{R}^{N} .
$$


Proof. Suppose the inequality does not hold. Then there exist $\eta_{0} \in \mathbf{R}^{N}$ and $\epsilon_{0}>0$ such that

$$
f\left(\lambda_{0}+\eta_{0}\right)<f\left(\lambda_{0}\right)-g^{\prime}\left(\lambda_{0}\right) \cdot \eta_{0}-\epsilon_{0} .
$$

Let $h(t)=f\left(\lambda_{0}+t \eta_{0}\right)-f\left(\lambda_{0}\right)+g^{\prime}\left(\lambda_{0}\right) \cdot t \eta_{0}$. Then $h$ is convex in $t \in \mathbf{R}$ and $h(0)=0, h(1)<-\epsilon_{0}$. By convexity, for all $0<t<1$,

$$
\frac{h(t)-h(0)}{t-0} \leq \frac{h(1)-h(0)}{1-0}
$$

and hence $h(t) \leq-\epsilon_{0} t$ for all $t \in(0,1)$. This implies

$$
f\left(\lambda_{0}+t \eta_{0}\right) \leq f\left(\lambda_{0}\right)-g^{\prime}\left(\lambda_{0}\right) \cdot t \eta_{0}-\epsilon_{0} t, \quad \forall t \in(0,1) .
$$

On the other hand, since $g$ is differentiable at $\lambda_{0}$, it follows that

$$
g\left(\lambda_{0}+t \eta_{0}\right)=g\left(\lambda_{0}\right)+g^{\prime}\left(\lambda_{0}\right) \cdot t \eta_{0}+o(t), \quad t \rightarrow 0^{+} .
$$

Therefore, we would have $f\left(\lambda_{0}+t \eta_{0}\right)+g\left(\lambda_{0}+t \eta_{0}\right)<f\left(\lambda_{0}\right)+g\left(\lambda_{0}\right)$ for all sufficiently small $t>0$. This contradicts the local minimality of $f+g$ at $\lambda_{0}$.

Proof of Theorem 2.8. Let $\bar{u}=\chi_{B} \bar{v}+\chi_{B^{c}} \bar{w}$, where $\bar{v}(x)=\bar{\lambda} \cdot x$ and $\bar{w}(x)=\frac{\bar{\lambda} \cdot x}{|x|^{N}}$. Then $\bar{u} \in X$ and we will show that $\bar{u}$ is a minimizer of $E^{\#}$. It is easy to show by elementary calculations that

$$
\Delta \bar{w}=0, \quad \frac{\partial \bar{w}}{\partial n}=(1-N) \bar{\lambda} \cdot x, \quad \int_{B^{c}}|\nabla \bar{w}|^{2}=|B|(N-1)|\bar{\lambda}|^{2} .
$$

Using the lemma above to $f=\psi^{\#}$ and $g=\frac{N-1}{2}|\lambda|^{2}$, we have

$$
\psi^{\#}(\bar{\lambda}+\eta) \geq \psi^{\#}(\bar{\lambda})-(N-1) \bar{\lambda} \cdot \eta, \quad \forall \eta \in \mathbf{R}^{N} .
$$

Then, for all $\zeta \in X$,

$$
\begin{aligned}
E^{\#}(\bar{u}+\zeta) & =\int_{B} \psi^{\#}(\bar{\lambda}+\nabla \zeta) d x+\frac{1}{2} \int_{B^{c}}|\nabla \bar{w}+\nabla \zeta|^{2} d x \\
& =\int_{B} \psi^{\#}(\bar{\lambda}+\nabla \zeta) d x+\frac{1}{2} \int_{B^{c}}|\nabla \bar{w}|^{2} d x+\int_{B^{c}} \nabla \bar{w} \cdot \nabla \zeta d x+\frac{1}{2} \int_{B^{c}}|\nabla \zeta|^{2} d x \\
& \geq \int_{B}\left[\psi^{\#}(\bar{\lambda})-(N-1) \bar{\lambda} \cdot \nabla \zeta\right] d x+\frac{N-1}{2}|\bar{\lambda}|^{2}|B|-\int_{\partial B} \frac{\partial \bar{w}}{\partial n} \zeta d S+\frac{1}{2} \int_{B^{c}}|\nabla \zeta|^{2} d x \\
& =|B|\left[\psi^{\#}(\bar{\lambda})+\frac{N-1}{2}|\bar{\lambda}|^{2}\right]+\frac{1}{2} \int_{B^{c}}|\nabla \zeta|^{2} d x \\
& =E^{\#}(\bar{u})+\frac{1}{2} \int_{B^{c}}|\nabla \zeta|^{2} d x .
\end{aligned}
$$

This proves that $E^{\#}(\bar{u})=|B|\left(\psi^{\#}(\bar{\lambda})+\frac{N-1}{2}|\bar{\lambda}|^{2}\right)$ is the minimum of $E^{\#}$. The remaining part on the necessary and sufficient condition for the existence of minimizers of $E$ follows from our Theorem 2.5 above and Theorem 1.1 in [19] (see also $[4,5]$ ). 


\section{The 2-dimensional soft ferromagnetism}

We will first apply our general principle to the simple case of two-dimensional soft ferromagnetism. Therefore, assume $N=2$ and $\varphi \equiv 0$. In this case, after some elementary calculus computations, the function $\Psi(\xi)$ defined by (1.13) above can be written as

$$
\Psi(\xi)=\min _{h \in S^{1}}\left[-(H+\xi) \cdot h+\frac{1}{2}\left(|\xi|^{2}+1\right)\right]=\frac{1}{2}(|\xi+H|-1)^{2}-\frac{1}{2}|H|^{2}-\xi \cdot H
$$

and the set $\sigma(\xi)$ is given by

$$
\sigma(-H)=S^{1} ; \quad \sigma(\xi)=\left\{\frac{H+\xi}{|H+\xi|}\right\} \quad \text { if } \xi \neq-H .
$$

The corresponding functional $E(u)$ defined by (1.16) above is thus

$$
E(u)=\int_{\mathbf{R}^{2}} \Phi(x, \nabla u(x)) d x=\int_{\Omega} \psi(\nabla u(x)) d x+\frac{1}{2} \int_{\Omega^{c}}|\nabla u(x)|^{2} d x
$$

where

$$
\psi(\lambda)=\Psi\left(\lambda^{\perp}\right)=\frac{1}{2}\left(\left|\lambda-H^{\perp}\right|-1\right)^{2}+\lambda \cdot H^{\perp}-\frac{1}{2}|H|^{2} .
$$

The convexification of $\psi$ can be easily computed and expressed in the form

$$
\psi^{\#}(\lambda)= \begin{cases}\frac{1}{2}\left(\left|\lambda-H^{\perp}\right|-1\right)^{2}+\lambda \cdot H^{\perp}-\frac{1}{2}|H|^{2} & \text { if }\left|\lambda-H^{\perp}\right| \geq 1, \\ \lambda \cdot H^{\perp}-\frac{1}{2}|H|^{2} & \text { if }\left|\lambda-H^{\perp}\right| \leq 1 .\end{cases}
$$

Note that $\psi^{\#}$ is in fact $C^{1}$ and

$$
\left(\psi^{\#}\right)^{\prime}(\lambda)= \begin{cases}\lambda-\frac{\lambda-H^{\perp}}{\left|\lambda-H^{\perp}\right|} & \text { if }\left|\lambda-H^{\perp}\right| \geq 1 \\ H^{\perp} & \text { if }\left|\lambda-H^{\perp}\right| \leq 1\end{cases}
$$

The detachment set for this function $\psi$ is $\mathcal{D}=\left\{\left|\lambda-H^{\perp}\right|<1\right\}$, on which the convexification $\psi^{\#}$ is affine. By Corollary 2.7, we easily have the following existence result.

Theorem 3.1 In the two-dimensional soft case, the energy $E$ and hence the energy I always have minimizers. Moreover a minimizer $\bar{m}$ of $I$ is given in the form of $\bar{m}(x)=\frac{H+\nabla^{\perp} \bar{u}(x)}{\left|H+\nabla^{\perp} \bar{u}(x)\right|}$ for a.e. $x \in \Omega$, where $\bar{u}$ is a minimizer of $E$.

However, even in this concrete case, finding a minimizer of $E$ may be a very difficult problem as the necessary and sufficient condition for minimizers illustrated by condition (2.11) is difficult to study. By (2.11c), only functions $u \in X$ satisfying $\left|\nabla u(x)-H^{\perp}\right| \geq 1$ for almost every $x \in \Omega$ can be a minimizer of energy $E$.

We will examine two special cases. 


\subsection{The case of small external applied fields}

First of all, we focus on functions $u \in X$ that satisfy

$$
\left|\nabla u(x)-H^{\perp}\right|=1 \quad \text { a.e. } \quad x \in \Omega .
$$

For all such functions, the third condition in (2.4) always holds, and the second and fourth conditions in (2.4) reduce to the exterior Neumann boundary problem:

$$
\Delta u=0 \text { in } \Omega^{c}, \quad \frac{\partial u}{\partial n}=H^{\perp} \cdot n \text { on } \partial \Omega .
$$

This problem has a unique solution $u=w_{H} \in X_{2}$, which also depends linearly on $H$. In fact, let $w_{i} \in X_{2}(i=1,2)$ be the unique solution of

$$
\Delta w_{i}=0 \text { in } \Omega^{c}, \quad \frac{\partial w_{i}}{\partial n}=n_{i} \text { on } \partial \Omega,
$$

where $n=\left(n_{1}, n_{2}\right)$, as always, is the outward unit normal to the interior domain $\Omega$. Then the solution $u=w_{H}$ of the Neumann problem (3.2) above can be written as

$$
w_{H}=-h_{2} w_{1}+h_{1} w_{2}=H^{\perp} \cdot\left(w_{1}, w_{2}\right) \text { in } \Omega^{c} .
$$

Let $u_{i} \in X$ be an extension of $w_{i}$ to whole $\mathbf{R}^{2}(i=1,2)$ and define

$$
u_{H}=-h_{2} u_{1}+h_{1} u_{2}=H^{\perp} \cdot\left(u_{1}, u_{2}\right) \quad \text { in } \mathbf{R}^{2} .
$$

Define $\tilde{g}=\left.w_{H}\right|_{\partial \Omega} \in Y$. Under the condition (3.1), this $\tilde{g}$ will be the unique boundary data $\bar{g}$ as determined in the general setting above. However, equation (3.1) has to be combined with the boundary condition

$$
\left.u\right|_{\partial \Omega}=\tilde{g}=\left.w_{H}\right|_{\partial \Omega} .
$$

We assume that $\Omega$ is sufficiently smooth so that $u_{i} \in X \cap C^{1}\left(\mathbf{R}^{2}\right)$. It is well known ([8]) that Problem (3.1), (3.3) admits (infinitely many) solutions if

$$
\left|\nabla u_{H}-H^{\perp}\right| \leq 1 \quad \text { in } \Omega .
$$

We have thus proved the following

Proposition 3.2 If condition (3.4) holds, then the minimizers of $E$ are given by

$$
u=\chi_{\Omega} v+\chi_{\Omega^{c}} w_{H},
$$

where $w_{H} \in X_{2}$ is the unique solution of problem (3.2) and $v$ is any function satisfying

$$
\left|\nabla v(x)-H^{\perp}\right|=1 \quad \text { in } \Omega,\left.\quad v\right|_{\partial \Omega}=\left.w_{H}\right|_{\partial \Omega} .
$$

In particular, when there is no applied field: $H=0$, then the distance function $v(x)=\operatorname{dist}(x, \partial \Omega)$ provides a minimizer $u=\chi_{\Omega}(x) \operatorname{dist}(x, \partial \Omega)$.

Note that

$$
\left|\nabla u_{H}(x)-H^{\perp}\right|=\left|h_{1}\right|\left|\nabla u_{2}(x)-(0,1)\right|+\left|h_{2}\right|\left|\nabla u_{1}(x)-(1,0)\right| \leq C|H|, \quad x \in \Omega,
$$

where $C$ is a constant depending only on the domain $\Omega$. Therefore, condition (3.4) is always satisfied if $|H|$ is sufficiently small. 


\subsection{The case of unit disk}

Let $\Omega=B$, the unit disc in $\mathbf{R}^{2}$. By Theorem 2.8 and its Remark, the unique boundary data $\bar{g}=\bar{\lambda} \cdot x$ and the corresponding exterior harmonic function $\omega(\bar{g})(x)=\frac{\bar{\lambda} \cdot x}{|x|^{2}}$, where $\bar{\lambda} \in \mathbf{R}^{2}$ is the unique solution of

$$
\left(\psi^{\#}\right)^{\prime}(\bar{\lambda})+\bar{\lambda}=0 .
$$

Solving this equation yields that $\bar{\lambda}=-H^{\perp}$ if $|H| \leq \frac{1}{2}$ and $\bar{\lambda}=-\frac{H^{\perp}}{2|H|}$ if $|H| \geq \frac{1}{2}$. Therefore, by Theorem 2.5, we have the following

Proposition 3.3 (i) If $|H|<\frac{1}{2}$, then the minimizers of $E$ are given non-uniquely by

$$
u=\chi_{B}(x) v(x)-\chi_{B^{c}}(x) \frac{H^{\perp} \cdot x}{|x|^{2}},
$$

where $v$ is any function satisfying

$$
\left|\nabla v(x)-H^{\perp}\right|=1 \quad \text { in } B,\left.\quad v\right|_{\partial B}=-H^{\perp} \cdot x .
$$

(ii) If $|H| \geq \frac{1}{2}$, then the (unique) minimizer of $E$ is given by

$$
\bar{u}=-\chi_{B}(x) \frac{H^{\perp} \cdot x}{2|H|}-\chi_{B^{c}}(x) \frac{H^{\perp} \cdot x}{2|H||x|^{2}} .
$$

\section{The 2-dimensional hard ferromagnetism}

\subsection{The uniaxial case}

As mentioned in the introduction, we assume the anisotropy density $\varphi$ is given by

$$
\varphi(m)=\beta(1-|m \cdot e|),
$$

where $\beta>0$ and $e \in S^{1}$ are given. In this case, we can easily compute the function $\Psi$ defined above as follows.

$$
\begin{aligned}
\Psi(\xi) & =\min _{h \in S^{1}}\left[\beta-|\beta e \cdot h|-(H+\xi) \cdot h+\frac{1}{2}\left(|\xi|^{2}+1\right)\right] \\
& =\frac{1}{2}\left(|\xi|^{2}+1\right)+\beta-\max \{|\xi+H+\beta e|,|\xi+H-\beta e|\} \\
& =\frac{1}{2}\left(|\xi|^{2}+1\right)+\beta-\max _{ \pm}\{|\xi+H \pm \beta e|\} .
\end{aligned}
$$

In this case, the density function $\psi$ is given by

$$
\psi(\lambda)=\Psi\left(\lambda^{\perp}\right)=\frac{1}{2}\left(|\lambda|^{2}+1\right)+\beta-\max _{ \pm}\left\{\left|\lambda-(H \pm \beta e)^{\perp}\right|\right\} .
$$


Let

$$
E(u)=\int_{\Omega} \psi(\nabla u(x)) d x+\frac{1}{2} \int_{\Omega^{c}}|\nabla u(x)|^{2} d x .
$$

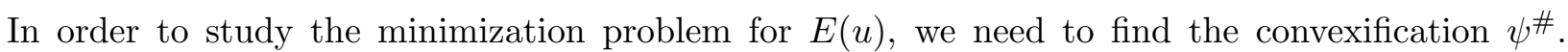
Writing $\lambda=t e^{\perp}+s e+H^{\perp}$ with $(t, s) \in \mathbf{R}^{2}$, we can express $\psi(\lambda)$ in terms of $(t, s)$ as follows.

$$
\psi(\lambda)=h(t, s)+\left(e^{\perp} \cdot H^{\perp}\right) t+\left(e \cdot H^{\perp}\right) s+\beta+\frac{1+|H|^{2}}{2},
$$

where the nonlinear part $h(t, s)$ is given by

$$
h(t, s)=\frac{1}{2}\left(t^{2}+s^{2}\right)-\sqrt{(|t|+\beta)^{2}+s^{2}} .
$$

It suffices to compute the convexification $h^{\#}$ of $h$ on $\mathbf{R}^{2}$. Note that $h$ satisfies

$$
h(-t,-s)=h(t,-s)=h(-t, s)=h(t, s), \quad(t, s) \in \mathbf{R}^{2} .
$$

We can restrict the computation to the first quadrant $Q=\{t \geq 0, s \geq 0\}$. Note that on $Q$

$$
h_{t}(t, s)=t-\frac{t+\beta}{\sqrt{(t+\beta)^{2}+s^{2}}}, \quad h_{s}(t, s)=s-\frac{s}{\sqrt{(t+\beta)^{2}+s^{2}}} .
$$

We want to know where $h(t, s)$ is increasing regarding $t$ and $s$ individually. So consider the domain $\Gamma \subset Q$ defined by $h_{t}(t, s) \geq 0$ and $h_{s}(t, s) \geq 0$. Then $\Gamma=\left\{(t, s) \in Q \mid t \sqrt{(t+\beta)^{2}+s^{2}} \geq t+\beta\right\}$. Write $\Gamma=\{(t, s) \in Q \mid s \geq 0, \gamma(s) \leq t<\infty\}$, where $t=\gamma(s), s>0$, is the inverse function of function

$$
s=\sigma(t)=\frac{t+\beta}{t} \sqrt{1-t^{2}}, \quad 0<t \leq 1 .
$$

Easy calculations show that $h(t, s)$ is convex on $\Gamma$ and increasing on $t$ and on $s$. Let $\delta(s)=h(\gamma(s), s)$ for $s \geq 0$. Then $\delta(s)$ is $C^{\infty}$ on $s \geq 0$ and $\delta^{\prime}(s)=h_{s}(\gamma(s), s)>0$ for $s>0$, and hence $\delta$ is increasing on $s \geq 0$. We now define a function $\tilde{h}$ on $Q$ by

$$
\tilde{h}(t, s)= \begin{cases}h(\gamma(s), s), & s \geq 0,0 \leq t \leq \gamma(s) \\ h(t, s), & s \geq 0, \gamma(s) \leq t<\infty\end{cases}
$$

Then it follows easily that $\tilde{h}$ is convex on $Q$ and nondecreasing in each of $t \geq 0$ and $s \geq 0$. We extend $\tilde{h}(t, s)$ onto $(t, s) \in \mathbf{R}^{2}$ according to the property

$$
\tilde{h}(-t,-s)=\tilde{h}(t,-s)=\tilde{h}(-t, s)=\tilde{h}(t, s), \quad(t, s) \in \mathbf{R}^{2} .
$$

Then this new function $\tilde{h}$ is convex on $\mathbf{R}^{2}$ and we have

Proposition 4.1 The convexification $h^{\#}$ of $h$ is equal to the extended function $\tilde{h}$. Therefore, the convexification $\psi^{\#}$ is given by $\psi^{\#}(\lambda)=\tilde{h}(t, s)+\left(\lambda-H^{\perp}\right) \cdot H^{\perp}+\beta+\frac{1+|H|^{2}}{2}$ and satisfies

$$
\left(\psi^{\#}\right)^{\prime}(\lambda)=\tilde{h}_{t}(t, s) e^{\perp}+\tilde{h}_{s}(t, s) e+H^{\perp},
$$

where $\lambda=t e^{\perp}+s e+H^{\perp}$. 
Remark. Note that $\psi^{\#}$ is not affine on the detachment set $\mathcal{D}=\left\{\lambda \mid \psi^{\#}(\lambda)<\psi(\lambda)\right\}$ and hence we cannot apply Corollary 2.7 above. In fact, in this case, the minimizers may not exist. For example, assume $H=0$. Then $\left(\psi^{\#}\right)^{\prime}(0)=0$; hence, by $(2.4)$ above, the unique boundary data $\bar{g}=0$. Moreover, any minimizer $\bar{u}$ of $E$ must satisfy

$$
\nabla \bar{u}(x) \in\left\{e^{\perp},-e^{\perp}\right\} \text { a.e. } x \in \Omega ;\left.\quad \bar{u}\right|_{\partial \Omega}=0,
$$

which is certainly impossible. Therefore $E(u)$ does not have any minimizers for any domain $\Omega$ if $H=0$. This was shown initially in [12], and further pursued and explored in many other places (in particular in [7]).

However, for some other $H$, the minimizers may exist. We study the special case for unit disk. In this case, to determine the boundary data $\bar{g}$, we need to solve equation: $\left(\psi^{\#}\right)^{\prime}(\lambda)+\lambda=0$. Writing $H^{\perp}=a e^{\perp}+b e$ and using (4.1) above, this equation is equivalent to

$$
\tilde{h}_{t}(t, s)+t+2 a=0, \quad \tilde{h}_{s}(t, s)+s+2 b=0 .
$$

The solution $(\bar{t}, \bar{s})$ is uniquely determined by $(a, b)$ and hence by $H$. Define the set

$$
\mathcal{H}=\left\{H \in \mathbf{R}^{2} \mid h(\bar{t}, \bar{s})>\tilde{h}(\bar{t}, \bar{s})\right\} .
$$

Then, it is easy to see that the line $\{s e \mid s \in \mathbf{R}\} \subset \mathcal{H}$ and the half rays $\left\{t e^{\perp}|| t \mid \geq \frac{1}{2}\right\} \subset \mathbf{R}^{2} \backslash \mathcal{H}$. Hence $\mathcal{H} \subsetneq \mathbf{R}^{2}$.

Proposition 4.2 Let $\Omega=B$ be the unit disk. Then the uniaxial ferromagnetic energy $I(m)$ has (unique) minimizer if $H \notin \mathcal{H}$ and has no minimizers if $H \in \mathcal{H}$.

Proof. Since $\tilde{h}$ and hence $\psi^{\#}$ are not affine on any open sets, by Theorem 2.8, $E$ and hence $I$ have a minimizer if and only if $h(\bar{t}, \bar{s})=\tilde{h}(\bar{t}, \bar{s})$. This proves the result.

Remark. This result says that if the applied field is in the direction of the easy axis then there are no global minimizers, but the preferred magnetizations can easily form microstructure to minimize the total energy. If the applied field is large and orthogonal to the easy direction, then forming microstructure will cost more energy and the global energy minimizer exists.

\subsection{The bi-axial case}

For the cubic (biaxial) situation we will put

$$
\varphi(m)=\beta \min \left\{1-|m \cdot e|, 1-\left|m \cdot e^{\perp}\right|\right\},
$$

where $e$ is a unit vector pointing in the direction of one of the easy axes. Pursuing the same computations as in the uniaxial case with the same notation, one finds the expression

$$
\psi(\lambda)=h(t, s)+\left(e^{\perp} \cdot H^{\perp}\right) t+\left(e \cdot H^{\perp}\right) s+\beta+\frac{1+|H|^{2}}{2},
$$


where the nonlinear part $h(t, s)$ is given by

$$
h(t, s)=\frac{1}{2}\left(t^{2}+s^{2}\right)-\max \left\{\sqrt{(|t|+\beta)^{2}+s^{2}}, \sqrt{(|s|+\beta)^{2}+t^{2}}\right\} .
$$

In addition to the same symmetries that we previously had in the uniaxial case, we also have that $h(t, s)=h(s, t)$. We can calculate that the detachment set is the region bounded by curves $h_{t}(t, s)=h_{s}(t, s)$ and also that the convexification $\tilde{h}$ of $h$ is constant $(=\min h)$ on the square $Q=\{|t|+|s| \leq 1\}$ and is affine only on each line segment of $|t|+|s|=c, c \geq 1$, between the mentioned curves.

Again, we consider the case when $\Omega=B$ is the unit disk. Let $\bar{\lambda}=\bar{t} e^{\perp}+\bar{s} e+H^{\perp}$ be the solution of $\left(\psi^{\#}\right)^{\prime}(\bar{\lambda})+\bar{\lambda}=0$ with $(\bar{t}, \bar{s})$ uniquely determined by $H$, and define the following sets

$$
\mathcal{H}_{1}=\left\{H \in \mathbf{R}^{2} \mid h(\bar{t}, \bar{s})=\tilde{h}(\bar{t}, \bar{s})\right\}, \quad \mathcal{H}_{2}=\left\{H \in \mathbf{R}^{2} \mid(\bar{t}, \bar{s}) \in Q\right\} .
$$

Then, we have the following existence and non-existence results.

Proposition 4.3 Let $\Omega=B$ be the unit disk. Then the biaxial ferromagnetic energy $I(m)$ has a unique minimizer if $H \in \mathcal{H}_{1}$, has infinitely many minimizers if $H \in \mathcal{H}_{2}$ and has no minimizers if $H \notin \mathcal{H}_{1} \cup \mathcal{H}_{2}$.

Proof. Since the open set where $\tilde{h}$ is affine is only the square $\left\{(t, s) \in \mathbf{R}^{2}|| t|+| s \mid<1\right\}$, by Theorem 2.8, $E$ and hence $I$ have a minimizer if and only if either $h(\bar{t}, \bar{s})=\tilde{h}(\bar{t}, \bar{s})$ or $|\bar{t}|+|\bar{s}|<1$. The first case we have the unique minimizer and the second case we have infinitely many minimizers. This proves the result.

Acknowledgement This contribution started while the first author was visiting Michigan State University during the fall of 2006. Its hospitality and support he would like to acknowledge.

\section{References}

[1] R. A. Adams and J. Fournier, "Sobolev Spaces," Second Edition. Academic Press, New York, 2003.

[2] J. M. Ball, A. Taheri and M. Winter, Local minimizers in micromagnetics and related problems, Calc. Var., 14 (2002), 1-27.

[3] P. Celada, G. Cupini and M. Guidorzi, A sharp attainment result for nonconvex variational problems, Calc. Var., 20 (2004), 301-328.

[4] A. Cellina, On minima of a functional of the gradient: necessary conditions, Nonlinear Anal., 20 (1993), 337-341.

[5] A. Cellina, On minima of a functional of the gradient: sufficient conditions, Nonlinear Anal., 20 (1993), 343-347. 
[6] R. Choksi and R. V. Kohn, Bounds on the micromagnetic energy of a unixial ferromagnet, Comm. Pure Appl. Math., LI (1998), 259-289.

[7] B. Dacorogna and I. Fonseca, A-B quasiconvexity and implicit partial differential equations, Calc. Var., $14(2)$ (2002), 115-149.

[8] B. Dacorogna and P. Marcellini, General existence theorems for Hamilton-Jacobi equations in the scalar and vectorial cases, Acta Math., 178(1) (1997), 1-37.

[9] A. DeSimone, R. V. Kohn, S. Müller and F. Otto, A reduced theory for thin-film micromagnetics, Comm. Pure and Appl. Math., LV (2002), 1408-1460.

[10] A. DeSimone, R. V. Kohn, S. Müller, F. Otto and R. Schäfer, Two-dimensional modelling of soft ferromagnetic films, Proc. R. Soc. Lond. A, 457 (2001), 2983-2991.

[11] G. Gioia and R. D. James, Micromagnetics of very thin films, Proc. R. Soc. Lond. A, 453 (1997), 213-223.

[12] R. D. James and D. Kinderlehrer, Frustration in ferromagnetic materials, Cont. Mech. Thermodyn., 2 (1990), 215-239.

[13] M. Kruzík and A. Prohl, Recent developments in the modeling, analysis, and numerics of ferromagnetism, SIAM Review, 48(3) (2006), 439-483.

[14] P. Pedregal, Relaxation in ferromagnetism: the rigid case, J. Nonlinear Science, 4 (1994), $105-125$.

[15] P. Pedregal, "Parametrized Measure and Variational Principles." Birkhauser, Basel, 1997.

[16] P. Pedregal, Relaxation in magnetostriction, Calc. Var., 10 (2000), 1-19.

[17] T. Riviére and S. Serfaty, Limiting domain wall energy for a problem related to micromagnetics, Comm. Pure and Appl. Math., LIV (2001), 294-338.

[18] T. Roubícek and M. Kruzík, Mesoscopic model for ferromagnets with isotropic hardening, $Z$. angew Math. Phys., 56 (2005), 107-135.

[19] M. A. Sychev, Characterization of homogeneous scalar variational problems solvable for all boundary data, Proc. Roy. Soc. Edinburgh, Sect A 130 (2000), 611-631. 